\title{
Estimating Approximate Incentive Compatibility
}

\author{
MARIA-FLORINA BALCAN, TUOMAS SANDHOLM, and ELLEN VITERCIK, Carnegie Mellon \\ University
}

In practice, most mechanisms for selling, buying, matching, voting, and so on are not incentive compatible. We present techniques for estimating how far a mechanism is from incentive compatible. Given samples from the agents' type distribution, we show how to estimate the extent to which an agent can improve his utility by misreporting his type. We do so by first measuring the maximum utility an agent can gain by misreporting his type on average over the samples, assuming his true and reported types are from a finite subset-which our technique constructs-of the type space. The challenge is that by measuring utility gains over a finite subset of the type space, we might miss pairs of types $\theta$ and $\hat{\theta}$ where an agent with type $\theta$ can greatly improve his utility by reporting the type $\hat{\theta}$. Our technique discretizes the type space by constructing a learning-theoretic cover in a higher-dimensional space. The key technical contribution is proving that the maximum utility gain over this finite subset nearly matches the maximum utility gain overall, despite the volatility of the utility functions we study. We apply our tools to the single-item and combinatorial first-price auctions, generalized second-price auction, discriminatory auction, uniform-price auction, and second-price auction with spiteful bidders. To our knowledge, these are the first guarantees for estimating approximate incentive compatibility from the mechanism designer's perspective.

CCS Concepts: • Theory of computation $\rightarrow$ Sample complexity and generalization bounds; Algorithmic game theory and mechanism design.

Additional Key Words and Phrases: Incentive compatibility, mechanism design, strategyproof, auctions, machine learning, learning theory, approximate incentive compatibility

ACM Reference Format:

Maria-Florina Balcan, Tuomas Sandholm, and Ellen Vitercik. 2019. Estimating Approximate Incentive Compatibility. In ACM EC '19: ACM Conference on Economics and Computation (EC '19), June 24-28, 2019, Phoenix, AZ, USA. ACM, New York, NY, USA, 1 page. https://doi.org/10.1145/3328526.3329628

Acknowledgments. The authors thank Zhe Feng, Noah Golowich, Harikrishna Narasimhan, and David Parkes for valuable discussion.

This material is based on work supported by the National Science Foundation under grants IIS-1718457, IIS-1617590, CCF-1733556, CCF-1422910, CCF-1535967, and IIS-1618714 the ARO under award W911NF-17-1-0082, an Amazon Research Award, a Microsoft Research Faculty Fellowship, a Google Research Award, and a National Science Foundation Graduate Research Fellowship.

The full draft of this paper can be found at: https://arxiv.org/pdf/1902.09413.pdf.

Permission to make digital or hard copies of part or all of this work for personal or classroom use is granted without fee provided that copies are not made or distributed for profit or commercial advantage and that copies bear this notice and the full citation on the first page. Copyrights for third-party components of this work must be honored. For all other uses, contact the owner/author(s).

EC '19, June 24-28, 2019, Phoenix, AZ, USA

(C) 2019 Copyright held by the owner/author(s).

ACM ISBN 978-1-4503-6792-9/19/06

https://doi.org/10.1145/3328526.3329628 